\title{
Voting on pensions: Sex and marriage
}

\author{
Marie-Louise Leroux ${ }^{\mathrm{a}, *}$, Pierre Pestieau ${ }^{\mathrm{b}}$, María Racionero ${ }^{\mathrm{c}}$ \\ a CORE, Université catholique de Louvain, B-1348 Louvain-la-Neuve, Belgium \\ b CORE, Université catholique de Louvain, B-1348, and CREPP, University of Liege, B-4000, Belgium \\ c Research School of Economics, Australian National University, Canberra, Australia
}

\section{A R T I C L E I N F O}

\section{Article history:}

Received 10 September 2009

Received in revised form 1 October 2010

Accepted 13 October 2010

Available online $\mathrm{xxxx}$

\section{JEL classification:}

D72

D78

H55

\section{Keywords:}

Social security

Majority voting

Derived rights

Individualisation of pension rights

\begin{abstract}
A B S T R A C T
Existing political economy models of pensions focus on age and productivity. In this paper we incorporate two additional individual characteristics: sex and marital status. We ignore the role of age, by assuming that people vote at the start of their life, and characterize the preferred rate of taxation that finances a Beveridgean pension scheme when individuals differ in wage, sex and marital status. We allow for two types of couples: one-breadwinner and two-breadwinner couples. Marriage pools both wage and longevity differences between men and women. Hence singles tend to have more extreme preferred tax rates than couples. We show that the majority voting outcome depends on the relative number of one-breadwinner couples and on the size of derived pension rights.
\end{abstract}

(c) 2010 Elsevier B.V. All rights reserved.

\section{Introduction}

Most pension schemes provide benefits that are longevity-invariant and sometimes contribution-invariant. Given that men have a shorter life expectancy than women and earn, and thus contribute, more, it is clear that such pension systems are to their detriment. So why do men consistently agree with pension schemes that penalize them? The first answer one can offer is that women outnumber men and can impose their views. Another possible reason is that, with flat-rate benefits, low-income men may support such schemes if earnings differences dominate longevity differences. Yet the best alternative explanation might be that, in a society where a majority of men and women are married, longevity and earnings differences are pooled within the couple and this makes any sex war irrelevant.

Women live longer than men and they earn less than men on average. For instance, in France, it is estimated that women life expectancy at 60 is $20 \%$ higher than that of a man and that the pay gap is around $20 \%$. At the same time, there is evidence that lowincome people, men and women, have lower longevity than high-income people. This has led to studies that show that social security schemes that look redistributive, but provide longevity-invariant benefits, are in fact not so redistributive (see e.g. Coronado et al., 2000; Liebman, 2001, and Bommier et al., 2006). For example, Bommier et al. (2006) estimate that the redistribution in the French public pension system is reduced by up to 50\% because of this longevity invariance.

Social Security redistribution by marital status is also surprisingly large. For instance, Galasso (2002) shows that one-earner couples get the highest internal return from the Social Security, followed by two-earner couples with 70/30 earnings split; returns are equal for two-earner couples with a 50/50 earnings split and single women, while single men are the most disadvantaged. The

\footnotetext{
* Corresponding author. Tel.: + 3210 478257; fax: + 3210474301.

E-mail address: marie-louise.leroux@uclouvain.be (M.-L. Leroux).
} 
difference in returns observed between singles and married couples, either one-earner or two-earner, can be explained by the socalled "derived pension rights". Several countries, like France, provide the surviving spouse (more often the woman) with a survivor benefit, while some other countries provide one-earner couples with a higher replacement rate than the one applied to single men; some countries, like Belgium or Japan, provide both types of derived benefits. ${ }^{1}$ The marital status and the generosity of the system towards the non-working spouse are then likely to play an important role in the support for a pension system.

A number of political economy papers have attempted to explain existing pension systems using majority voting models. ${ }^{2}$ In his seminal contribution, Browning (1975) focused on age differences and showed that, if the old favour generous pensions and the young prefer private savings, the decisive voter is the median age one. More recent models include wage differences alongside age differences. In such a framework, Casamatta et al. (2000) show that the pension system is chosen by a majority made of rich and poor workers who collude against a coalition of retirees and middle class workers: this is the so-called ends against the middle outcome.

In this paper we concentrate on two additional individual characteristics: sex and marital status. We characterize the pension scheme that is chosen by majority voting in a society where men live shorter and earn more than women, and most men and women are married. Assuming that retirement consumption is financed by the returns of private savings and a Beveridgean pension benefit, we explore several issues: i) the effect of longevity and wage gender gaps on the chosen tax rate, ii) the effect of an increase in the number of married couples on the size of the pension system, iii) the effect of an increase in the relative number of one-breadwinner (versus two-breadwinner) couples on the pension system, and iv) the effect of the individualization of pension rights (equivalently, the reduction of derived pension rights) on the size of the chosen tax rate.

These issues are certainly relevant for prevailing pension systems and, surprisingly, have hardly been addressed in the literature. ${ }^{3}$ In particular, the generosity of the system towards non-working spouses may play an important role in the political support of one-breadwinner couples towards existing pension systems. ${ }^{4}$ This is a timely topic to address. Indeed, women are increasingly participating in the labour force and pension systems are increasingly individualized. More and more countries are abandoning the "derived pension rights model" and adopting instead the so-called "adult worker model". For example, Denmark has suppressed survivor benefits and Germany has moved towards a "family splitting" system, which also provides a compensation for interrupted careers (for example, a pension credit per child). ${ }^{5}$ One can thus expect that this dual evolution (i.e. increased labour participation by women and individualization of pension rights) will have some incidence on the size of the pension system.

The setting we adopt is standard. People live for two periods, the second one being of variable length. They work in the first period and retire in the second one. The retirement consumption depends on the amount of private savings but also on the pension benefit, which is chosen through voting. Finally, the pension system is Beveridgean so that pension benefits and payroll tax rates are uniform. Individuals vote at the beginning of their life. To keep the analysis tractable, we make a number of simplifying assumptions like, for instance, a quasi-linear utility function, no liquidity constraints and certain length of life. All men have the same longevity, which is lower than that of women. Men and women have the same productivity, but the wage of women is only a fixed fraction of that of men. Later in the paper, we discuss the implications of assuming a continuous productivity distribution instead. We further assume that the number of singles and of couples with one or two earners is exogenously given. ${ }^{6}$ In this model, there is also positive assortative mating (i.e. men marry women who have the same underlying productivity but earn a given fraction of their wage) ${ }^{7}$

In this framework, lower productivity and higher longevity individuals benefit from the existence of a pension scheme. Thus, single women, who have lower wages and longer lives, will be in favour of a pension scheme while single men, who have higher wages and shorter lives, will be against it. We then explore the role of couples. We first consider two-breadwinner couples only. In this case gender differences in wages and longevity are neutralized so that the couple gets a zero net benefit from the pension system. They are hence indifferent between public pensions and private savings as a mean of smoothing consumption between periods. However, because labour supply is endogenous in our setting, a pension system creates labour supply distortions and that they end up preferring a zero tax rate. Finally we account for the existence of one-breadwinner couples, alongside singles and twobreadwinner couples, and introduce derived pension rights. One-breadwinner couples do not neutralize gender differences and they will be in favour of a generous pension system when derived pension rights are important and sufficient to outweigh the husband's net contribution to the pension system. We show that the support for a pension system depends on the relative proportions of different types of households, and on the size of the derived pension rights. We further extend our model to allow for a continuous productivity distribution. Our results are robust to this new specification. The only difference is that now, for each

\footnotetext{
${ }^{1}$ For example, in Belgium, the supplementary pension is evaluated to $1 / 4$ of the working spouse pension. As shown in Gruber and Wise (1999), derived pension rights may take very different forms depending on the country.

2 For good surveys, see Galasso and Profeta (2002) and de Walque (2005).

3 See however Borck (2007) and Leroux (2010) who have introduced longevity differentials in political economy models of social security.

${ }^{4}$ For a good survey on the role of derived pension rights on old-age income security of women in OECD countries, see Choi (2006).

5 On this, see Choi (2006), Veil (2007) and Bonnet and Geraci (2009).

6 This is certainly not what is observed in reality. For instance, the introduction of a pension system, providing or not derived rights may modify the marital structure of the society and may also influence the women's labour participation decision. For instance, in Leroux and Pestieau (2010), it depends on the features of pension systems.

7 The papers of Mare (1991), Pencavel (1998) and Qian (1998) find strong evidence of positive assortative mating with respect to education. Education can be regarded as a good proxy for income.
} 
type of household, some individuals (the ones at the bottom of the productivity distribution) are always in favour of a pension system due to the amount of income redistribution they obtain.

Finally, our model suggests that the recent trend towards the individualization of pension rights should lead to reduced payroll tax levels. Our model however does not provide clear results regarding the effect of an increase in the proportion of twobreadwinner couples, where an increase or decrease in the preferred tax rate are both possible depending on the generosity of the system.

The rest of the paper is organized as follows. Section 2 presents a standard political economy model where individuals differ in wages and longevity. Section 3 introduces gender and marriage. In Section 4 we allow for productivity differences and in the last section we discuss the assumptions made in our model and some possible extensions.

\section{The basic model}

We assume that individuals live for two periods. ${ }^{8}$ They work in the first period and retire in the second one. Each individual of type $i$ is characterized by a pair $\left(w_{i}, \pi_{i}\right)$, where $w_{i}$ is the labour productivity in the first period and $\pi_{i}$ is the length of the second period of life. ${ }^{9}$

The intertemporal utility function of any individual of type $i$ is quasi-linear (linear in the first-period consumption) and is represented by

$$
u_{i}\left(c_{i}, d_{i}, l_{i}\right)=c_{i}-v\left(l_{i}\right)+\pi_{i} u\left(d_{i}\right),
$$

where $c_{i}$ and $d_{i}$ denote the first- and second-period consumptions, respectively, and $l_{i}$ the is labour supply. Second-period utility function $u\left(\right.$.) is such that $u^{\prime}()>$.0 and $u^{\prime \prime}()<$.0 . For simplicity, we assume that the disutility of labour $v\left(l_{i}\right)$ is quadratic and equal to $l_{i}^{2} / 2$. Individuals work, contribute to the pension system, consume and save in the first period. In the second period, they retire and receive a pension benefit $p$. We also assume a perfect annuity market and a zero interest rate so that the return on savings is simply $1 / \pi_{i}$. First- and second-period consumptions can then be written as

$$
\begin{aligned}
c_{i} & =(1-\tau) w_{i} l_{i}-s_{i}, \\
d_{i} & =\frac{s_{i}}{\pi_{i}}+p,
\end{aligned}
$$

where $\tau \in[0,1]$ is the payroll tax rate and $s_{i}$ is the amount of savings. ${ }^{10}$

Throughout the paper, we assume away liquidity constraints so that $s_{i}$ can be positive as well as negative. ${ }^{11}$ The problem of type $i$ 's individual consists in solving

$$
\begin{aligned}
& \max _{l_{i}, c_{i}} \quad c_{i}-l_{i}^{2} / 2+\pi_{i} u\left(d_{i}\right) \\
& \text { s.t. }\left\{\begin{array}{l}
c_{i}=(1-\tau) w_{i} l_{i}-s_{i} \\
d_{i}=\frac{s_{i}}{\pi_{i}}+p
\end{array}\right.
\end{aligned}
$$

From the first-order conditions, we obtain:

$$
\begin{aligned}
& l_{i}^{*}=(1-\tau) w_{i}, \\
& u^{\prime}\left(d_{i}^{*}\right)=1 .
\end{aligned}
$$

As to the pension benefit, we assume that individuals contribute to the pension system during the first period of their life and receive a flat pension benefit in the second period (i.e. the retirement period) ${ }^{12}$ Thus a feasible pension system must satisfy the budget constraint

$$
p \sum n_{i} \pi_{i} \leq \tau \sum n_{i} w_{i} l_{i}^{*},
$$

where $n_{i}$ denotes the relative number of individuals of type $i$. Note that here $p$ is an annual pension benefit, which implies that a

\footnotetext{
8 Our approach is static but our model can equivalently be seen as the steady state of an open economy OLG model.

9 An alternative would be to allow for uncertain mortality, $\pi_{i}$ being then the probability of surviving to the second period. We believe that it would not modify substantially our conclusions but would complicate the presentation.

${ }^{10}$ Note that second-period consumption $d_{i}$ and, accordingly, its components (i.e. the private annuity and the pension benefit) represent instant flows during a period of unequal length $\pi_{i}$.

11 Under this assumption, we allow for negative first-period consumption. This simplifies our results without changing them qualitatively.

12 Assuming a pension benefit that would be partially contributive would not change the nature of the results but would complicate the analysis.
} 


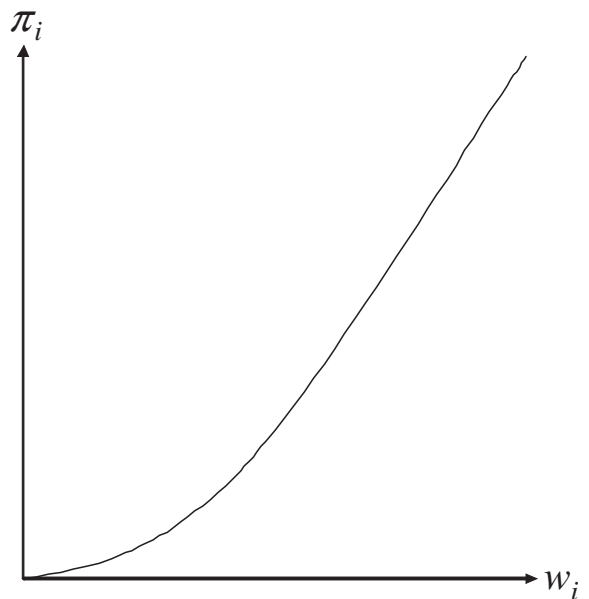

Fig. 1. Separating locus of types for and against a positive payroll tax.

person that lives longer gets more in total than a person that has a shorter life. Under the assumption of perfect budget balance, the expression for the pension benefit is

$$
p(\tau)=\tau \frac{(1-\tau) E\left[w^{2}\right]}{\bar{\pi}},
$$

where $E\left[w^{2}\right]$ is the average square wage and $\bar{\pi}$ is the average length of the second period. ${ }^{13}$ Every individual contributes an amount that is proportional to his labour income and receives a uniform pension benefit during a retirement period of unequal length $\pi_{i}$. Such a pension system redistributes resources from high-productivity to low-productivity individuals and from shortlived to long-lived individuals. ${ }^{14}$

The indirect utility function of an individual of type $i$ is then

$$
V^{i}(\tau)=\frac{(1-\tau)^{2} w_{i}^{2}}{2}-s_{i}^{*}+\pi_{i} u\left(\frac{s_{i}^{*}}{\pi_{i}}+p(\tau)\right)
$$

where the star stands for the optimal level. The preferred tax rate of this individual is obtained by solving the following program:

$$
\max _{\tau \in[0,1]} V^{i}(\tau) .
$$

In Appendix A we show that the solution to this problem is

$$
\tau_{i}^{*}=\left\{\begin{array}{l}
0 \text { for } \frac{w_{i}^{2}}{\pi_{i}} \geqslant \frac{E\left[w^{2}\right]}{\bar{\pi}}, \\
\frac{E\left[w^{2}\right]}{\bar{\pi}}-\frac{w_{i}^{2}}{\pi_{i}} \\
\frac{E\left[w^{2}\right]}{\bar{\pi}}-\frac{w_{i}^{2}}{\pi_{i}}
\end{array}\right. \text { otherwise. }
$$

The preferred tax rate of an individual depends on the level of redistribution he expects to get from the pension system. He may benefit from the pension system either because of a longer life and/or of a lower productivity than the average. Hence, the preferred tax rate of any individual will be zero if he has characteristics such that $w_{i}^{2} / \pi_{i} \geqslant E\left[w^{2}\right] / \bar{\pi}$. It is clear that the lower the wage rate and the higher the longevity the more likely an individual will be in favour of the pension scheme. The equality $w_{i}^{2} / \pi_{i}=E\left[w^{2}\right] / \bar{\pi}$ gives the separating locus of types for and against a positive payroll tax and, thus, a public pension scheme. In Fig. 1, we represent this function in the plane $\left(w_{i}, \pi_{i}\right)$.

To the left of the curve, the $\left(w_{i}, \pi_{i}\right)$-types are in favour of a positive tax; to the right, they are against. It is also worth noticing that, when positive, the most preferred tax rate decreases with $w_{i}$ and increases with $\pi_{i}$.

${ }^{13}$ Note that in this section the terms "wage" and "productivity" can be used interchangeably. This will not be the case in subsequent sections and we use then different notation to distinguish them.

${ }^{14}$ Most PAYG pension schemes exhibit such features. On this topic see, for example, Coronado et al. (2000), Liebman (2001) and Bommier et al. (2006). 
For someone with a zero wage, the most preferred tax rate is equal to $1 / 2$, and not 1 . This is due to the efficiency cost of taxation: $1 / 2$ is the tax that provides the maximum revenue (i.e. the peak of the Laffer curve).

The determination of the political equilibrium when voters differ in more than one characteristic is known to be more complex. ${ }^{15}$ We address this below by assuming a particular relationship between the characteristics $w_{i}$ and $\pi_{i}$.

\section{Model with unique productivity level}

We now assume that individuals differ in gender. We consider first a society consisting only of singles, and introduce later the possibility of marriage. We also allow couples to comprise either one breadwinner or two breadwinners.

We assume that there is a mass 1 of men as well as of women. These are characterized by a pair $\left(\pi_{m}, \omega_{m}\right)$ for men and a pair $\left(\pi_{f}, \omega_{f}\right)$ for women such that

$$
\begin{aligned}
& \pi_{m}=\pi, \omega_{m}=w, \\
& \pi_{f}=\beta \pi, \omega_{f}=\alpha w,
\end{aligned}
$$

where $\omega_{i}$ represents the wage, which might differ from productivity $w$ for women. We assume that $\alpha \leq 1$ and $\beta \geq 1 .{ }^{16}$ In other words, we posit that women have a longer life than men but obtain a lower wage. Note that, in this section, we assume a unique productivity level. ${ }^{17}$ In this case, the pension benefit (Eq. (1)) is now equal to

$$
p(\tau)=\tau(1-\tau) \frac{\left(1+\alpha^{2}\right) w^{2}}{(1+\beta) \pi},
$$

since the average expected wage is now $\left(1+\alpha^{2}\right) w^{2} / 2$ and the average longevity is $\bar{\pi}=(1+\beta) \pi / 2$.

\subsection{The political equilibrium in a society of singles}

Under our assumptions of different wage and longevity for different genders, using Eq. (3), we have that the preferred tax rates for men and women are, respectively,

$$
\begin{array}{ll}
\tau_{m}^{*}=0 & \text { since } \quad \frac{w^{2}}{\pi} \geq \frac{\left(1+\alpha^{2}\right) w^{2}}{(1+\beta) \pi}, \\
\tau_{f}^{*}>0 & \text { since } \frac{\alpha^{2} w^{2}}{\beta \pi} \geq \frac{\left(1+\alpha^{2}\right) w^{2}}{(1+\beta) \pi} .
\end{array}
$$

A man, who has lower longevity and higher productivity than the average, always prefers a zero tax rate since he is a net contributor to the pension system. On the contrary, a woman always gets a net benefit from the pension system and votes for a positive tax rate

$$
\tau_{f}^{*}=\frac{\beta-\alpha^{2}}{\beta-\alpha^{2}+\beta\left(1+\alpha^{2}\right)}>0 .
$$

The political equilibrium corresponds to the preferred tax rate of the median individual. For instance, if the number of women was slightly higher than the number of men, the political outcome in a society composed by singles only would be the preferred tax rate of women:

$$
\tau^{*}=\tau_{f}^{*} .
$$

\subsection{The political equilibrium in a society with both singles and couples}

We now study the labour and consumption decisions made by a couple. Note that we do not consider endogenous marriage even if, obviously, some single individuals may gain from forming a couple. Indeed, given the quasi-linear specification, the utility of a couple is the same as the sum of utilities of a single man and a single woman with the same productivity. From a laissez-faire perspective, marriage creates a welfare gain for the woman and a loss for the man, which is reduced when there is a pension

\footnotetext{
15 The determination of the equilibrium when preferences are single-peaked and individuals differ in a single characteristic is relatively simpler. For instance, if all individuals had the same longevity $\pi_{i}=\bar{\pi}$, the equilibrium tax rate would depend on the productivity distribution. Assume for example a standard density function with median wage below average wage: $\bar{w} \geq w_{\text {med }}$. We know from Jensen inequality that $\sqrt{E\left[w^{2}\right]}>\bar{w}$. Given that in the relevant range of $w$, the most preferred tax rate decreases with $w$, the Condorcet winner is the tax rate preferred by the individuals with median wage.

${ }^{16}$ For simplicity, we restrict attention to the most realistic case where $\alpha \leq 1$ and $\beta \geq 1$ but the analysis could be extended to $\alpha>1$ and $\beta<1$. We do not explore in this paper why women have a lower wage. We simply account for the empirical fact that women, on average, have a lower wage than men.

${ }^{17}$ In Section 4, we relax this assumption to allow for a continuous productivity distribution.
} 
system. There may be also other reasons why individuals get married or not, which we do not model here. We believe that it would complicate the model without changing our qualitative results. ${ }^{18}$ Additionally, in this paper we adopt the unitary model of the household (i.e. a model where the household has only one set of preferences). ${ }^{19}$ Under such a specification, spouses play cooperatively and share their resources over their life-cycle. A two-breadwinner couple thus solves the following problem:

$$
\begin{aligned}
& \max _{c, d, l_{m}, l_{f}} 2 c-l_{f}^{2} / 2-l_{m}^{2} / 2+\left(\pi_{f}+\pi_{m}\right) u(d) \\
& \text { s.t. }\left(\omega_{m} l_{m}+\omega_{f} l_{f}\right)(1-\tau)+\left(\pi_{f}+\pi_{m}\right) p \geq 2 c+\left(\pi_{f}+\pi_{m}\right) d
\end{aligned}
$$

where $d$ represents the individual level of (annual) consumption in the second period for each member of the couple. The labour supply of the husband and that of the wife are, respectively, $l_{m}^{*}=w(1-\tau)$ and $l_{f}^{*}=\alpha w(1-\tau)$. Note that, under our assumptions, these are independent of their marital status (i.e. whether they belong to a couple or are single). Hence, the labour supply of a woman is always lower than that of a man. This implies that her total contribution to the pension scheme, $\alpha w \tau$, is also lower while she receives a higher total pension benefit, $\beta \pi p \geq \pi$.

Substituting for $l_{m}^{*}$ and $l_{f}^{*}$ and $p(\tau)$, we obtain the couple's indirect utility function

$$
V^{c 2}(\tau)=\frac{(1-\tau)^{2}}{2}\left(1+\alpha^{2}\right) w^{2}+(1+\beta) \pi\left[u\left(d^{*}\right)-d^{*}+p(\tau)\right],
$$

where the superscript $c 2$ stands for a couple with two breadwinners and $d^{*}$ is the optimal level of second-period consumption. ${ }^{20}$ The equation of the pension benefit (Eq. (4)) is not modified by the introduction of two-breadwinner couples since both members contribute to, and benefit from, the pension scheme in the same way as if they were singles. Differentiating this indirect utility function with respect to the tax rate $\tau$, it is straightforward to show that the preferred tax rate of a two-breadwinner couple is always nil, $\tau_{c 2}^{*}=0$. Note that, if labour supply were exogenous, the couple would be indifferent between any level of taxation (it would obtain the same return from savings as from the pension scheme). When labour supply is endogenous, the preferred tax rate is zero since, in this case, the pension scheme introduces distortions on the labour supply (i.e. the individual return from the pension scheme is smaller than the return from private savings).

We now consider the political equilibrium and assume that a fraction $\varphi$ of men and women are married. The preferred tax rates for single women and men remain the same, since the existence of couples does not modify the expression of the pension benefit, so that $\tau_{m}^{*}=0$ and $\tau_{f}^{*}>0$ (as shown before) while $\tau_{c 2}^{*}=0$. With equal number of women and men, as soon as some of them are married there is a majority of individuals who favour a zero tax rate and the political outcome will be $\tau^{*}=0 .{ }^{21}$

\subsection{Introducing one-breadwinner couples}

\subsubsection{The modified model}

Let us now assume that society consists of four different categories of households: single men, single women, couples with two breadwinners and couples with one breadwinner. As in the previous sections, there is still an equal fraction $(1-\varphi)$ of single males and of single females and a fraction $\varphi$ of couples, so that a number $2 \varphi$ of individuals live in couple. But we now assume that, among these couples, a fraction $\mu$ is composed of two breadwinners, while a fraction $(1-\mu)$ of couples consists of only one breadwinner. ${ }^{22}$

The earner is always the husband. ${ }^{23}$ His wife may be entitled to a pension benefit in the retirement period even if she did not personally contribute to the pension scheme. These benefits, sometimes called derived pension rights, consist of a small supplementary pension plus a survival pension. We thus assume that she receives a fraction $\gamma \in[0,1]$ of the full annual pension benefit $p(\tau)$ during the second period of her life of length $\pi_{f}{ }^{24}$ If $\gamma=0$ the spouse receives nothing in the second period while if $\gamma=1$ she gets a full pension. ${ }^{25}$ Whatever the value of $\gamma$, annual consumption is the same for both spouses.

\footnotetext{
${ }^{18}$ In equilibrium, the pension benefit depends on the structure of the society (through the government budget constraint), which itself depends on the pension parameters. This phenomenon is exacerbated with the introduction of one-breadwinner couples and derived rights. For simplicity, we assume that the number of married individuals is exogenous. Although very interesting, we believe that endogenizing marriage is outside the scope of the paper and we left this for future work.

${ }^{19}$ A number of alternatives have been recently suggested, ranging from bargaining to non-cooperative models. Our choice is mainly guided by the concern for simplicity.

20 The quasi-linearity of first period consumption implies that we assume away income effects on $d^{*}$ and $l^{*}$.

21 If the labour supply were exogenous, the couple would be indifferent between any level of taxation and we would then have had exactly the reverse result, i.e. a maximum tax rate $\tau^{*}=\tau_{f}^{*}=1$, under the assumption of a slightly higher number of women than of men.

22 Note that Section 3.1 is equivalent to assuming $\varphi=0$, while Section 3.2 corresponds to $\varphi \in[0,1]$ and $\mu=1$.

${ }^{23}$ Up to now, the total lifetime income was $Y=w^{2}\left(1+\alpha^{2}\right)$, normalizing the size of the population to 2 . With a fraction $\varphi(1-\mu)$ of the population as onebreadwinner couples, this amount decreases to $Y^{\prime}=w^{2}\left[1+\alpha^{2}(1-\varphi(1-\mu))\right]$.

${ }^{24}$ The parameter $\gamma$ may account either for a survivor benefit or for the higher replacement rate provided to a one-earner couple than to a single individual. As mentioned in the introduction, such features are observed in many countries with a public pension scheme.

${ }^{25}$ Note that we assume that $\gamma$ is exogenously fixed while, in reality, it is certainly a political choice variable. In the present paper we make this assumption in order to simplify our computations and to focus on the influence of the structure of the society on the size of the pension system (through the tax rate). This assumption is relaxed in Leroux and Pestieau (2010) where it is assumed either that individuals vote on the tax rate and that $\gamma$ is optimally fixed at a constitutional level.
} 
We also assume that the female participation decision is exogenous. We do so as this decision may depend on many factors such as personal ones that we do not model here (like child bearing and rearing, etc.) but also on the pension design. Since this decision is correlated with the form and the size of the pension rights given to the non-working spouse, which in turn depend also on the marital and labour force structure of society (through the government budget constraint), for simplicity we assume that the woman decision to work is exogenous and the segmentation between one- and two-breadwinner couples is fixed. ${ }^{26}$

Let us first explore the problem of a one-breadwinner couple, which is slightly different from the two-breadwinner one (i.e. problem $A)$ :

$$
\begin{aligned}
& \max _{c, d, l_{m}} 2 c-l_{m}^{2} / 2+\left(\pi_{f}+\pi_{m}\right) u(d) \\
& \text { s.t. } \omega_{m} l_{m}(1-\tau)+\left(\gamma \pi_{f}+\pi_{m}\right) p(\tau) \geq 2 c+\left(\pi_{f}+\pi_{m}\right) d
\end{aligned}
$$

Only the man supplies labour, with $l_{m}^{*}=w(1-\tau)$. Substituting for $l_{m}^{*}$ and $\pi_{f}$, the indirect utility function is equal to

$$
V^{c 1}(\tau)=\frac{(1-\tau)^{2} w^{2}}{2}+(1+\gamma \beta) \pi p(\tau)+(1+\beta) \pi\left[u\left(d^{*}\right)-d^{*}\right]
$$

where the superscript $c 1$ stands for a couple with one breadwinner.

The expression for $p(\tau)$ is now modified due to the existence of one-breadwinner couples. To see this clearly, we rewrite the budget constraint as

$$
p[\pi+\beta \pi(1-\varphi+\varphi \mu+\gamma \varphi(1-\mu))] \leq \tau\left[w l_{m}^{*}+\alpha w l_{f}^{*}(1-\varphi+\varphi \mu)\right]
$$

On the left-hand side we have total benefits distributed: every working individual (men or women) receives a pension $p$ and the fraction $\varphi(1-\mu)$ of non-working women receive $\gamma p$. On the right-hand side we have total contributions. In this model men supply labour, whatever their marital status, but only women who are single or belong to two-breadwinner couples supply labour and thus pay contributions. Using the optimal labour supplies, total contributions are equal to $(1-\tau) \tau\left[1+\alpha^{2}(1-\varphi+\varphi \mu)\right] w^{2}$. Then, the new expression for the flat-rate pension benefit is:

$$
p(\tau)=\tau \frac{(1-\tau)\left[1+\alpha^{2}(1-\varphi+\varphi \mu)\right] w^{2}}{[1+\beta(1-\varphi+\varphi \mu+\gamma \varphi(1-\mu))] \pi} .
$$

Note that if we assume a society of only singles $(\varphi=0)$, or a society with two-breadwinner couples $(\varphi \in(0,1]$ and $\mu=1), p(\tau)$ is equal to Eq. (4) as before and we recover the solutions obtained in the previous subsections.

For the following sections, and in order to simplify notation, we define here the function $\chi(\alpha, \beta, \varphi, \mu, \gamma)$ :

$$
\chi(\alpha, \beta, \varphi, \mu, \gamma)=\frac{1+\alpha^{2}(1-\varphi+\varphi \mu)}{1+\beta(1-\varphi+\varphi \mu+\gamma \varphi(1-\mu))} .
$$

The "modified" pension benefit can be rewritten as

$$
p(\tau)=\tau(1-\tau) \frac{w^{2}}{\pi} \chi(\alpha, \beta, \varphi, \mu, \gamma)
$$

\subsubsection{Preferred tax rates and the political equilibrium}

Individuals preferred tax rates are obtained by solving

$$
\max _{\tau \in[0,1]} V^{i}(\tau)
$$

where $i$ accounts for $m$ (single male), $f$ (single female), $c 2$ (two-breadwinner couples) and $c 1$ (one-breadwinner couples). For onebreadwinner couples, the indirect utility function is Eq. (6) while, for the other households, indirect utility functions remain the same and given by Eqs. (2) and (5); only the expression for $p(\tau)$ is modified and given by Eq. (8). In Appendix B, we derive the solution for each type of individual and show that the preferred tax rates are equal to:

$$
\tau_{m}^{*}=0
$$

\footnotetext{
26 In the paper by Leroux and Pestieau (2010) the society comprises only couples, and the tax rate and the size of derived rights are chosen both through voting and at the constitutional level. In that case the labour supply decision of the wife depends both on a housework cost and on the pension design (Bismarckian or Beveridgean with derived pension rights).
} 


$$
\begin{aligned}
& \tau_{f}^{*}=\left\{\begin{array}{l}
0 \quad \text { if } \gamma>\hat{\gamma}_{f} \equiv \frac{\beta-\alpha^{2}}{\beta \alpha^{2} \varphi(1-\mu)} \\
\frac{\beta \chi(\alpha, \beta, \varphi, \mu, \gamma)-\alpha^{2}}{2 \beta \chi(\alpha, \beta, \varphi, \mu, \gamma)-\alpha^{2}} \quad \text { if } \gamma<\hat{\gamma}_{f}
\end{array}\right. \\
& \tau_{c 1}^{*}= \begin{cases}0 \quad \text { if } \gamma<\hat{\gamma}_{c} \equiv \frac{\beta-\alpha^{2}}{\beta\left(1+\alpha^{2}\right)} \\
\frac{(1+\beta \gamma) \chi(\alpha, \beta, \varphi, \mu, \gamma)-1}{2(1+\beta \gamma) \chi(\alpha, \beta, \varphi, \mu, \gamma)-1} \quad \text { if } \gamma>\hat{\gamma}_{c}\end{cases} \\
& \tau_{c 2}^{*}= \begin{cases}0 \quad \text { if } \gamma \geq \hat{\gamma}_{c} \\
\frac{(1+\beta) \chi(\alpha, \beta, \varphi, \mu, \gamma)-\left(1+\alpha^{2}\right)}{2(1+\beta) \chi(\alpha, \beta, \varphi, \mu, \gamma)-\left(1+\alpha^{2}\right)} & \text { if } \gamma<\hat{\gamma}_{c}\end{cases}
\end{aligned}
$$

where $\hat{\gamma}_{f}$ and $\hat{\gamma}_{c}$ are threshold levels of $\gamma$. Above $\hat{\gamma}_{f}$ single women prefer a zero tax and below $\hat{\gamma}_{f}$ they prefer a positive tax rate. Below $\hat{\gamma}_{c}$, one-breadwinner couples prefer a zero tax and above $\hat{\gamma}_{c}$, they prefer a positive tax rate. The threshold level for twobreadwinner couples $\hat{\gamma}_{c}$ coincides with that of one-breadwinner couples, but the interests of these two types of households are opposed, with two-breadwinner couples preferring a zero tax rate above $\hat{\gamma}_{c}$ and positive tax rates below $\hat{\gamma}_{c}$. As before, the preferred tax rates of single men are zero. Their preference for a zero tax rate is here reinforced by the fact that the pension system now also redistributes towards one-breadwinner couples. For all other types of households, the level of preferred tax rate depends on the value of $\gamma$ (i.e. the level of generosity of the pension scheme towards one-breadwinner couples). Single women prefer a strictly positive tax rate only when the system is not too generous towards the non-working spouse, since more redistribution to the latter is to the detriment of single women (they get less from the pension scheme). For one-breadwinner couples, we obtain the opposite: they will prefer a strictly positive tax rate if the scheme is sufficiently redistributive towards them. If $\gamma \rightarrow 0$, the treatment one-breadwinner couples receive is similar to that of males. In this specific case, the couple obtains almost no survivor or dependent benefit compensation and they vote for a zero tax rate. On the contrary, if $\gamma$ is high the husband's contribution to the pension scheme can be compensated by the benefit received by his non-working spouse. Two-breadwinner couples tend to prefer zero tax rates unless the generosity of the system towards one-breadwinner couples is sufficiently low. In this case the fact that both males and one-breadwinner couples are net contributors to the pension system can benefit two-breadwinner couples and they may vote for a positive tax despite its distortionary effects.

We now characterize the political equilibrium level of the tax rate. It depends on the generosity of the system towards onebreadwinner couples (i.e. on the level of $\gamma$ ) and the composition of the population (and particularly on $\mu$, i.e. the proportion of twobreadwinner couples). Note that $\hat{\gamma}_{c}<\hat{\gamma}_{f}$ (i.e., there is an interval where both $\tau_{f}^{*}$ and $\tau_{c 1}^{*}$ are positive). It is possible to show that

$$
\tau_{f}^{*}>\tau_{c 1}^{*} \quad \text { iff } \gamma<\frac{\beta-\alpha^{2}}{\beta \alpha^{2}} \equiv \hat{\gamma}
$$

and that $\hat{\gamma} \in\left[\hat{\gamma}_{c}, \hat{\gamma}_{f}\right]$. It is also possible to show that $\tau_{f}^{*}>\tau_{c 2}^{*}$ within the interval where both are positive (i.e. $\left.0<\gamma<\hat{\gamma}_{c}\right)$. This is not surprising since single females benefit from the pension system more than two-breadwinner couples regardless of the level of generosity. We obtain the following cases (see Appendix B for details):

- For low levels of generosity $\gamma<\hat{\gamma}_{c}$, preferred tax rates are $\tau_{m}^{*}=\tau_{c 1}^{*}=0, \tau_{c 2}^{*}>0$ and $\tau_{f}^{*}>0$, with $\tau_{f}^{*}>\tau_{c 2}^{*}$. If $\mu<1 / 2$, then $\tau^{*}=0$. If $\mu>1 /$ 2 , then $\tau^{*}=\tau_{c 2}^{*}>0$.

- For medium levels of generosity $\hat{\gamma}_{c}<\gamma<\hat{\gamma}_{f}$, preferred tax rates are $\tau_{m}^{*}=\tau_{c 2}^{*}=0, \tau_{c 1}^{*}>0$ and $\tau_{f}^{*}>0$. If $\mu>1 / 2$, then $\tau^{*}=0$. If $\mu<1 / 2$, then $\tau^{*}>0$. However, the value of the tax rate depends on the particular value of $\gamma$ with respect to $\hat{\gamma}$ (defined above). If $\hat{\gamma}_{c}<\gamma<\hat{\gamma}$, the preferred tax rate is $\tau_{c 1}^{*}$. If $\hat{\gamma}<\gamma<\hat{\gamma}_{f}$, the preferred tax rate is $\tau_{f}^{*}$.

- Finally, for high levels of generosity $\gamma>\hat{\gamma}_{f}$, only one-breadwinner couples vote for a positive tax rate, $\tau_{c 1}^{*}>0$, while all other categories vote for a zero tax rate. Hence, a zero tax rate results unless $\varphi(1-\mu)>1 / 2$ (i.e. the one-breadwinner couples form a majority). In that highly unlikely case, $\tau^{*}=\tau_{c 1}^{*}>0$.

To sum up, the existence of one-earner couples (and their relative proportion $1-\mu$ ), as well as the generosity of the system towards them (represented by $\gamma$ ), crucially influence the level of tax rate chosen by majority voting. A positive tax results for low levels of generosity when the proportion of two-breadwinner couples is more than half and that they vote alongside single women against the interests of single men and one-breadwinner couples, which prefer a zero tax rate as these are net contributors to the system. A positive tax results also for intermediate values of generosity when one-breadwinner couples represent a proportion $1-\mu>1 / 2$ and vote alongside single women for a positive tax rate as they are both net recipients from the pension system. However, the level of generosity needs to be relatively small so that single women still support it. If the level of generosity towards 
one-breadwinner becomes too large, single women withdraw their support and a positive tax would result only if onebreadwinner couples represent a majority in the population, which is highly unlikely.

In the next section, we extend our model to take into account differences in productivity, not only between genders but also across individuals in general.

\section{Model with continuous productivity distribution}

In this section we keep the assumption that longevity can take only two values (i.e. $\pi_{m}=\pi$ and $\pi_{f}=\beta \pi$ for men and women, respectively). In contrast, we now assume that productivity $w$ is uniformly distributed with support $[0,1]$. The average and the median productivity are thus identical and equal to $\bar{w}=w_{\text {med }}=1 / 2 .{ }^{27}$ Finally, we assume perfect assortative mating: when a man and a woman form a couple they do so with someone with the same underlying productivity, which means wages $w$ and $\alpha w$ for man and woman, respectively. ${ }^{28}$

With the results obtained above in mind, we expect that now every individual at the bottom of the wage distribution will be in favour of a pension system, since they will benefit from the inherent redistribution. Thus, independently of their marital status and gender, a fraction of individuals (in each type of household) will vote for a positive tax rate. In contrast, some individuals, those at the top of the wage distribution, will be against a pension system. In this section we study the relative support for a particular tax rate within each type of household.

\subsection{Preferred tax rates}

When productivity $w$ is uniformly distributed with support $[0,1]$ the pension benefit becomes

$$
p(\tau)=\frac{1}{3} \frac{\tau(1-\tau)}{\pi} \chi(\alpha, \beta, \varphi, \mu, \gamma)
$$

where $1 / 3$ corresponds to the average square productivity in the population. In Appendix $C$ we show that individuals' preferred tax rates are now:

$$
\begin{aligned}
& \tau_{f}^{*}(w)=\left\{\begin{array}{l}
0 \quad \text { if } \frac{\alpha^{2} w^{2}}{1 / 3}>\beta \chi(\alpha, \beta, \varphi, \mu, \gamma) \\
\frac{\beta}{3} \chi(\alpha, \beta, \varphi, \mu, \gamma)-\alpha^{2} w^{2} \\
2 \frac{\beta}{3} \chi(\alpha, \beta, \varphi, \mu, \gamma)-\alpha^{2} w^{2}
\end{array}\right. \text { otherwise } \\
& \tau_{m}^{*}(w)=\left\{\begin{array}{l}
0 \quad \text { if } \frac{w^{2}}{1 / 3}>\chi(\alpha, \beta, \varphi, \mu, \gamma) \\
\frac{\frac{\chi(\alpha, \beta, \varphi, \mu, \gamma)}{3}-w^{2}}{2 \frac{\chi(\alpha, \beta, \varphi, \mu, \gamma)}{3}-w^{2}} \quad \text { otherwise }
\end{array}\right. \\
& \tau_{c 2}^{*}(w)=\left\{\begin{array}{l}
0 \quad \text { if } \frac{w^{2}}{1 / 3}>\frac{(1+\beta)}{\left(1+\alpha^{2}\right)} \chi(\alpha, \beta, \varphi, \mu, \gamma) \\
\frac{\chi(\alpha, \beta, \varphi, \mu, \gamma)}{3}(1+\beta)-\left(1+\alpha^{2}\right) w^{2} \\
2 \frac{\chi(\alpha, \beta, \varphi, \mu, \gamma)}{3}(1+\beta)-\left(1+\alpha^{2}\right) w^{2}
\end{array}\right. \text { otherwise } \\
& \tau_{c 1}^{*}(w)=\left\{\begin{array}{l}
\frac{w^{2}}{1 / 3}>(1+\gamma \beta) \chi(\alpha, \beta, \varphi, \mu, \gamma) \\
\frac{\chi(\alpha, \beta, \varphi, \mu, \gamma)}{3}(1+\gamma \beta)-w^{2} \\
2 \frac{\chi(\alpha, \beta, \varphi, \mu, \gamma)}{3}(1+\gamma \beta)-w^{2}
\end{array}\right. \text { otherwise }
\end{aligned}
$$

where the $w$ relates to the productivity of the individuals belonging to the different groups $m, f, c 2$ and $c 1$.

\footnotetext{
27 We could alternatively assume a right-skewed distribution, but this would complicate our model without providing additional insights.

${ }^{28}$ As we mentioned previously, independently of their (low or high) productivity, some women do not work, which is due to exogenous reasons or preferences, which we do not model here. This is certainly an interesting extension (see Leroux and Pestieau, 2010).
} 


\subsection{Political equilibrium}

In order to characterize the political equilibrium, we manipulate expressions (13) to (16) so as to obtain the productivity as a function of the most preferred tax rate. With the uniform distribution assumption, this represents the proportion of individuals, within each type of household, who prefer this tax rate (or a greater one) to any other lower tax rate:

$$
\begin{aligned}
w_{m}(\tau) & =\sqrt{\frac{1-2 \tau}{1-\tau} \frac{\chi(\alpha, \beta, \varphi, \mu, \gamma)}{3}}, \\
w_{f}(\tau) & =\sqrt{\frac{\beta}{\alpha^{2}}} w_{m}(\tau), \\
w_{c 2}(\tau) & =\sqrt{\frac{1+\beta}{1+\alpha^{2}}} w_{m}(\tau), \\
w_{c 1}(\tau) & =\sqrt{1+\gamma \beta} w_{m}(\tau) .
\end{aligned}
$$

It is straightforward to show that $w_{m}(\tau)<w_{c 1}(\tau)$ and $w_{m}(\tau)<w_{c 2}(\tau)<w_{f}(\tau)$. In addition,

$$
\begin{aligned}
& w_{c 1}(\tau)>w_{f}(\tau) \text { iff } \gamma>\frac{\beta-\alpha^{2}}{\beta \alpha^{2}}, \\
& w_{c 1}(\tau)>w_{c 2}(\tau) \text { iff } \gamma>\frac{\beta-\alpha^{2}}{\beta\left(1+\alpha^{2}\right)} .
\end{aligned}
$$

We obtain three different patterns of support for a given tax rate $\tau$ :

$$
\begin{aligned}
& \text { Case }\left(\text { a) }: \gamma<\frac{\beta-\alpha^{2}}{\beta\left(1+\alpha^{2}\right)} \rightarrow w_{m}(\tau)<w_{c 1}(\tau)<w_{c 2}(\tau)<w_{f}(\tau),\right. \\
& \text { Case }\left(\text { b) }: \frac{\beta-\alpha^{2}}{\beta\left(1+\alpha^{2}\right)}<\gamma<\frac{\beta-\alpha^{2}}{\beta \alpha^{2}} \rightarrow w_{m}(\tau)<w_{c 2}(\tau)<w_{c 1}(\tau)<w_{f}(\tau),\right. \\
& \text { Case }(\text { c }): \gamma>\frac{\beta-\alpha^{2}}{\beta \alpha^{2}} \rightarrow w_{m}(\tau)<w_{c 2}(\tau)<w_{f}(\tau)<w_{c 1}(\tau)
\end{aligned}
$$

In case (a), for low levels of generosity $\gamma$, support from single women is larger, followed by two-breadwinner couples, then one-breadwinner couples and lastly single men. Note that at low levels of generosity the treatment of one-breadwinner couples is similar to the treatment of single men and it is then not surprising that their interests are more aligned. As the system becomes more generous towards one-breadwinner couples the pension system gains more support from this type of household than from two-breadwinner couples. Note that the threshold level of $\gamma$ that changes the pattern of support from case (a) to case (b) is precisely the threshold level $\hat{\gamma}_{c}$ we identified in the single productivity case. Finally, if the pension system becomes too generous towards one-breadwinner couples single women become less supportive. The threshold level of $\gamma$ associated with a switch from case (b) to case (c) is precisely the threshold level of $\hat{\gamma}$ we identified in the single productivity case.

We now turn to the characterization of the equilibrium payroll tax rate under majority voting. The equilibrium tax rate is defined such that at least one half of the population prefers this tax rate (or a higher one) to any other lower tax rate. The voting equilibrium tax rate $\tau^{*}$ is then such that the number of individuals with higher productivity (and thus who would prefer a lower tax rate) represents exactly one half of the total population:

$$
(1-\varphi)\left[w_{m}\left(\tau^{*}\right)+w_{f}\left(\tau^{*}\right)\right]+2 \varphi\left[\mu w_{c 2}\left(\tau^{*}\right)+(1-\mu) w_{c 1}\left(\tau^{*}\right)\right]=1,
$$

where a mass 1 of individuals corresponds to one half of the population. Solving the above equation (see Appendix C), we obtain that:

$$
\tau^{*}=\frac{\chi(\alpha, \beta, \varphi, \mu, \gamma) \Omega(\alpha, \beta, \varphi, \mu, \gamma)^{2}-3}{2 \chi(\alpha, \beta, \varphi, \mu, \gamma) \Omega(\alpha, \beta, \varphi, \mu, \gamma)^{2}-3}
$$

where

$$
\Omega(\alpha, \beta, \varphi, \mu, \gamma)=(1-\varphi)\left(1+\sqrt{\frac{\beta}{\alpha^{2}}}\right)+2 \varphi\left(\mu \sqrt{\frac{1+\beta}{1+\alpha^{2}}}+(1-\mu) \sqrt{1+\gamma \beta}\right) .
$$

We now illustrate this formula with a numerical example. We take as given $\alpha=0.8, \beta=1.2$ and $\varphi=0.6$ and we focus on the incidence of a variation in the proportion of two-breadwinner couples $(\mu)$ and in the generosity of the pension system $(\gamma)$ on the 


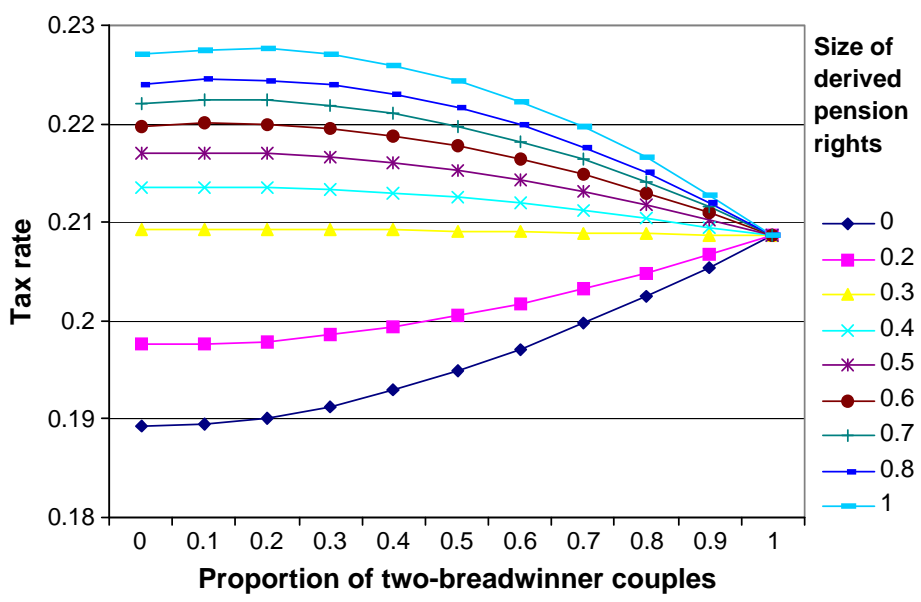

Fig. 2. Effect on the equilibrium tax rate of changes in the proportion of two-breadwinner couples and the size of derived pension rights.

equilibrium tax rate. ${ }^{29}$ These changes relate to the two phenomena already mentioned: increasing labour participation of women and individualization of pension systems. The results are reported in Fig. 2.

For any level of $\mu<1$, the equilibrium tax rate is increasing in $\gamma$ (i.e. in the generosity towards one-breadwinner couples). The analytical expression for $\partial \tau^{*} / \partial \gamma$ (see Appendix C) depends on two effects: $\partial \chi(.) / \partial \gamma$ and $\partial \Omega(.) / \partial \gamma$. The term $\chi($.$) is a factor of$ proportionality in the annual pension benefits that stems from the budget constraint and is related to the ratio of contributions to payments. We show that $\partial \chi(.) / \partial \gamma<0$ : an increase in $\gamma$, ceteris paribus, decreases the annual pension since a given amount of contributions is shared among a larger proportion of individuals. The term $\Omega\left(\right.$.) stems from the net political support condition. ${ }^{30}$ We show that $\partial \Omega(.) / \partial \gamma>0$. The combined effect, according to the expression for $\partial \tau^{*} / \partial \gamma$, is a priori ambiguous. We find however a condition for $\partial \tau^{*} / \partial \gamma>0$ that depends exclusively on the parameters of the model. This analytical expression (in Appendix C) allows us to carry out robustness checks on the numerical simulations. We are able to show that the condition holds for a large range of reasonable parameter values. ${ }^{31}$ As the generosity towards one-breadwinner couples increases, and we move from case (a) to case (c) characterized above, the pattern of support for the pension system changes, increasing among one-breadwinner couples and decreasing among the rest. However, for the range of parameters investigated, the combined effect leads to a positive effect of the size of derived pension rights on the equilibrium tax rate.

In contrast, the variation of the tax rate with $\mu$ (i.e. the number of two-breadwinner couples) is ambiguous and depends on the level of $\gamma$. In Appendix $\mathrm{C}$ we provide the analytical expression for $\partial \tau^{*} / \partial \mu$, which depends also on $\partial \chi(.) / \partial \mu$ and $\partial \Omega(.) / \partial \mu$. The sign of these expressions depends now on the value of $\gamma$ : we show that $\partial \chi(.) / \partial \mu>0$ and $\partial \Omega(.) / \partial \mu<0$ when $\gamma>\hat{\gamma}_{c}$, whereas $\partial \chi($.) $/ \partial \mu<0$ and $\partial \Omega(.) / \partial \mu>0$ for $\gamma<\hat{\gamma}_{c}$. To understand the effect of $\mu$ on $\chi($.) note that the increase in the proportion of working spouses affects both sides of the budget: as more married women work, the tax base increases (see footnote 23) but they also become eligible to full pensions. The expenditure on derived pension rights diminishes as the proportion of non-working spouses decreases. However, the relative importance of this effect depends on the level of generosity of the system: if $\gamma$ is high, increasing the proportion of working spouses substantially decreases the amount spent on derived pension rights, and the combined effect on $\chi($.$) is positive, whereas if \gamma$ is low the decrease in amount paid in derived pension rights and the increase in tax receipts are not enough to compensate for the increased outlays in full pensions to working spouses, and the combined effect on $\chi($.$) is negative.$ To understand the effect of changes on $\mu$ on $\Omega($.$) , which is associated with the political support for the pension system, it is worth$ recalling that $w_{c 1}(\tau)<w_{c 2}(\tau)$ if and only if $\gamma<\hat{\gamma}_{c}$. This low level of generosity corresponds to case (a) described above where the support for the pension system is primarily driven by single women and two-breadwinner couples. An increase in $\mu$ increases the political support. On the contrary, $\gamma>\hat{\gamma}_{c}$, corresponds to cases (b) and (c) where support for the pension scheme is increasingly driven by one-breadwinner couples, the more so the larger $\gamma$ is. Hence, an increase in $\mu$, which decreases the proportion of onebreadwinner couples, leads to a decrease in the political support. For the particular parameter values of $\alpha$ and $\beta$ used, the threshold value of generosity is $\hat{\gamma}_{c}=0.2845$. In our illustration it appears that the political support effect outweighs the budget effect: when

\footnotetext{
${ }^{29}$ The values for $\alpha, \beta$ and $\varphi$ are close to the ones observed in France. It is estimated that the pay gap between men and women within the same socioprofessional category is around $19 \%$, while the life expectancy at 60 of a woman is around $21 \%$ higher than the one of men. It is also the case that the division of households between singles and couples is such that $59 \%$ of households are couples (See the website of the French national institute for economic studies and statistics, www.insee.fr). As we mention in footnote 31, our results are robust to other (reasonable) specifications of $\alpha, \beta$ and $\varphi$.

${ }^{30}$ We call it net support because in $\Omega(\alpha, \beta, \varphi, \mu, \gamma), w_{m}(\tau)$ does not appear.

${ }^{31}$ Note that this result holds for any value $\mu \in[0,1], \varphi \in[0,1], \gamma \in[0,1]$ and also when $\beta$ increases. Only when $\alpha$, which represents the proportion of productivity that women earn, is sufficiently low $(\alpha \leq 0.4)$ the condition might fail to be fulfilled.
} 
$\gamma<0.2845$ the tax rate increases with the proportion of two-breadwinner couples whereas when $\gamma>0.2845$ we observe a decrease in the equilibrium tax rate as the proportion of one-breadwinner couples decreases.

The effects of changes in $\gamma$ and $\mu$ on the equilibrium tax rate, when both change simultaneously, may thus go in opposite directions. Hence, it is a priori impossible to predict unambiguously the effect on the pension system of the recent trends towards the increase in labour participation of married women and the individualization of pension rights. The model does however shed light on the underlying forces, and particularly on the changing political support as those parameters vary.

\section{Conclusion}

In this paper, we have introduced two individuals' characteristics - gender and marital status differences - that are not generally taken into account in political economy models of Social Security. Our analysis is one of the first to shed light on the importance of considering the couple as a distinct economic agent in order to explain the size of a pension system. ${ }^{32}$ As opposed to standard political economy models, which only consider single agents, we distinguish between single individuals, male or female, and couples. We also distinguish between one-breadwinner and two-breadwinner couples and account for the existence of derived pension rights.

We show that when there are only two wage levels (one for men and a lower one for women), only single men are always against the pension system. On the contrary, all the other categories (i.e. one-, two-breadwinner couples and single women) may favour it depending on the size of derived pension rights (i.e. the amount of redistribution one-breadwinner couples get from the pension system). On the one hand, women and two-breadwinner couples benefit from the redistribution operated by the pension system because of advantageous longevity and wage heterogeneity; on the other hand, one-breadwinner couples benefit from redistribution through derived pension rights. Thus, if derived pension rights are high, the pension system is favourable to onebreadwinner couples, but this is to the detriment of single women and two-breadwinner couples who may end up voting for a zero tax. We also generalize our analysis by assuming a continuous productivity distribution. We find that the equilibrium payroll tax decreases when the system becomes less generous towards one-breadwinner couples but the effect of changes in the relative number of two-breadwinner couples is found to be ambiguous.

Clearly, our paper shows that the marital status and the composition of households influence the political support for the pension system. Moreover, another important observation of our paper is that, while two-breadwinner couples neutralize gender differences in longevity and wage, one-breadwinner couples do not. If the pension system is generous towards the non-working spouses, these couples will push for large pension benefits. In that respect, it is worth noticing the current trend in many countries: the progressive decline in the proportion of couples with only one breadwinner and the individualization of pensions systems, which implies less generosity towards non-working spouses. According to our model, an increasing individualisation of the pension rights leads to a lower level of payroll taxation while the increasing number of two-breadwinner couples yields mitigated results. $^{33}$

In our paper we make a number of simplifying assumptions: no liquidity constraints, labour supply invariant to the marital status, zero interest rate, actuarially fair annuity, no widowers, quadratic disutility of labour, quasi-linear utility, uniform density of wages, assortative mating and no overlapping generations. We do not think that the qualitative results would change if these assumptions were relaxed; at the same time, it is clear that the analysis would be more complicated.

Finally, the type of pension system considered in this paper is the Beveridgean one: namely, a pension system wherein the annual pension is invariant to contributions and longevity. This does not mean that everyone has the same resources upon retirement. As public pensions are supplemented by private savings, it includes traditional savings but also all forms of defined contribution private pensions. An alternative specification would be the traditional Bismarckian system (i.e. a pension system in which benefits are related to earnings but independent of longevity). A further possibility would be a pension system in which pension benefits are related to both longevity and contributions. Such a system would be akin to private savings. The most realistic specification would probably include a mix of Beveridge and Bismarck with benefits partially related to contributions, with survival pension for spouses without or negligible pension rights, and minimum pensions. ${ }^{34}$

Another controversial aspect of our paper is that the level of derived pension rights is exogenously fixed. One could expect this to be a political choice variable. Assuming that individuals can vote both on the size of derived rights and on the tax rate level, but

\footnotetext{
32 The closest paper to ours is Recoules (2009), which focuses on the political support for family-friendly policies (i.e. policies aimed at child-rearing) in a society constituted only of couples and in which there is gender discrimination in the labour market. The degree of discrimination is likely to influence the size of government spending, under some conditions.

${ }^{33}$ There is a conjecture that the increasing participation of women in the labour market is accompanied by a decline in the level of wages. If this were the case the wealth effect would disappear and we would then expect that the increase in the number of two-breadwinner couples would lead to an unambiguous decrease in the equilibrium tax.

${ }^{34}$ A more realistic setting would have been to look at a pension system that would be a linear combination between a (Beveridgean) flat-rate benefit pension and a (Bismarckian) pension with benefits that would be related to both earnings and longevity and without derived rights. Under our assumptions (quasi-linear utility function and no liquidity constraints), the problem would be unchanged. The Bismarckian fraction would be identical to private saving and the vote would only concern the Beveridgean fraction along with the given derived rights. If the pension consisted of only the Bismarckian part, then there would not be any vote. Each individual would be indifferent between no pension and any level of Bismarckian pension, which would be neutralized by private saving.
} 
also allowing the structure of the society to be endogenous (i.e. to depend on the pension instruments) would certainly constitute an interesting extension. ${ }^{35}$

Our model could be extended in several other directions. First, we could investigate the implications of adjusting the couples' pension benefits for scale economies. Second, we have only considered differences in longevity between men and women but we have not accounted for the empirical fact that men have, on average, longer life expectancy when married than when single. Our conjecture is that, taking this second feature into account, would reinforce our results. The support for the pension system should increase as then, not only a married man would gain from the benefit received by his wife but also he would get benefit from a pension for a longer period than if he had been single.

\section{Acknowledgements}

The authors thank the participants to the CESifo area conference on the Public Sector 2009 in Munich, to the conference LouisAndré Gérard-Varet 2009 in Marseille, to the PET conference 2009 in Galway, to the Australasian Public Choice conference 2009 in Melbourne as well as two anonymous referees. Errors remain ours.

\section{A. Preferred tax rate}

We solve the following program:

$$
\max _{\tau \in[0,1]} V^{i}(\tau)=\frac{(1-\tau)^{2} w_{i}^{2}}{2}-s_{i}^{*}+\pi_{i} u\left(\frac{s_{i}^{*}}{\pi_{i}}+\tau(1-\tau) E\left[w^{2}\right] \bar{\pi}\right) .
$$

Differentiating $V^{i}(\tau)$ with respect to $\tau$, we obtain

$$
\frac{\partial V^{i}(\tau)}{\partial \tau}=-(1-\tau) w_{i}^{2}+\pi_{i} u^{\prime}\left(d_{i}^{*}\right) E\left[w^{2}\right] \bar{\pi}(1-2 \tau)
$$

with $u^{\prime}\left(d_{i}^{*}\right)=1$. Evaluating this expression at $\tau=0$, we find that any individual with $w_{i}^{2} / \pi_{i} \geq E\left[w^{2}\right] / \bar{\pi}$ always prefers a zero tax rate. For those with $w_{i}^{2} / \pi_{i} \geq E\left[w^{2}\right] / \bar{\pi}$, the solution is interior and the preferred tax rate is equal to Eq. (3).

\section{B. Model with unique productivity level}

Indirect utility functions of the four categories of household are

$$
\begin{aligned}
& V^{f}(\tau)=\frac{(1-\tau)^{2} \alpha^{2} w^{2}}{2}+\beta \pi\left[u\left(d^{*}\right)-d^{*}+p(\tau)\right] \\
& V^{m}(\tau)=\frac{(1-\tau)^{2} w^{2}}{2}+\pi\left[u\left(d^{*}\right)-d^{*}+p(\tau)\right] \\
& V^{c 1}(\tau)=\frac{(1-\tau)^{2} w^{2}}{2}+(1+\gamma \beta) \pi p(\tau)+(1+\beta) \pi\left[u\left(d^{*}\right)-d^{*}\right] \\
& V^{c 2}(\tau)=\frac{(1-\tau)^{2}}{2}\left(1+\alpha^{2}\right) w^{2}+(1+\beta) \pi d\left[u\left(d^{*}\right)-d^{*}+p(\tau)\right]
\end{aligned}
$$

with $p(\tau)$ given by Eq. (8).

Preferred tax rates are characterized by differentiating the indirect utility functions with respect to $\tau$ :

$$
\begin{aligned}
& \frac{\partial V^{f}(\tau)}{\partial \tau}=-(1-\tau) \alpha^{2} w^{2}+\beta \pi \frac{d p(\tau)}{d \tau}, \\
& \frac{\partial V^{m}(\tau)}{\partial \tau}=-(1-\tau) w^{2}+\pi \frac{d p(\tau)}{d \tau}, \\
& \frac{\partial V^{c 1}(\tau)}{\partial \tau}=-(1-\tau) w^{2}+(1+\gamma \beta) \pi \frac{d p(\tau)}{d \tau}, \\
& \frac{\partial V^{c 2}(\tau)}{\partial \tau}=-(1-\tau)\left(1+\alpha^{2}\right) w^{2}+(1+\beta) \pi \frac{d p(\tau)}{d \tau},
\end{aligned}
$$

\footnotetext{
${ }^{35}$ The paper by Leroux and Pestieau (2010) allows individuals to vote on the size of derived rights in a model where the structure of the society between oneand two-breadwinner couples varies with the features of the model (such as the design of the pension system and a housework cost).
} 
where

$$
\frac{d p(\tau)}{d \tau}=(1-2 \tau) \frac{w^{2}}{\pi} \chi(\alpha, \beta, \varphi, \mu, \gamma)
$$

with $\chi(\alpha, \beta, \varphi, \mu, \gamma)$ defined by Eq. (7).

Evaluating $\partial V^{i}(\tau) / \partial \tau$ at $\tau=0$, for $i=m, f, c 1, c 2$ we find that

$$
\begin{aligned}
& \left.\frac{\partial V^{m}(\tau)}{\partial \tau}\right|_{\tau=0}<0, \\
& \left.\frac{\partial V^{f}(\tau)}{\partial \tau}\right|_{\tau=0}<0 \text { iff } \frac{\beta-\alpha^{2}}{\beta \alpha^{2} \varphi(1-\mu)}<\gamma, \\
& \left.\frac{\partial V^{c 1}(\tau)}{\partial \tau}\right|_{\tau=0}<0 \text { iff } \gamma<\frac{\beta-\alpha^{2}}{\beta\left(1+\alpha^{2}\right)}, \\
& \left.\frac{\partial V^{c 1}(\tau)}{\partial \tau}\right|_{\tau=0}<0 \text { iff } \gamma>\frac{\beta-\alpha^{2}}{\beta\left(1+\alpha^{2}\right)},
\end{aligned}
$$

which imply the following threshold levels of $\gamma$ for single women, one-breadwinner and two-breadwinner couples, respectively:

$$
\begin{aligned}
& \hat{\gamma}_{f}=\frac{\beta-\alpha^{2}}{\beta \alpha^{2}} \frac{1}{\varphi(1-\mu)}, \\
& \hat{\gamma}_{c}=\frac{\beta-\alpha^{2}}{\beta\left(1+\alpha^{2}\right)},
\end{aligned}
$$

such that for $\gamma<\hat{\gamma}_{f}$, the preferred tax rate of a single woman is positive (resp. if $\gamma>\hat{\gamma}_{f}$, her preferred tax rate is zero), for $\gamma<\hat{\gamma}_{c}$ the preferred tax rate of a one-breadwinner couple is zero (resp. if $\gamma>\hat{\gamma}_{c}$, the preferred tax rate is positive) and for $\gamma<\hat{\gamma}_{c}$ the preferred tax rate is positive (resp. if $\gamma>\hat{\gamma}_{c}$, the preferred tax rate is zero). Thus, for $\gamma<\hat{\gamma}_{f}$, the preferred tax rate level of women is strictly positive and solves the following equality:

$$
-(1-\tau) \alpha^{2} w^{2}+\beta(1-2 \tau) \chi(\alpha, \beta, \varphi, \mu, \gamma) w^{2}=0
$$

This yields Eq. (10). We use the same procedure for one-breadwinner couples when $\hat{\gamma}_{c}>\gamma$ and for two-breadwinner couples when $\gamma<\hat{\gamma}_{c}$. The solutions are interior and yield Eqs. (11) and (12), respectively.

It is easy to show that $\hat{\gamma}_{c}<\hat{\gamma}_{f}$ since

$$
\frac{\beta-\alpha^{2}}{\beta\left(1+\alpha^{2}\right)}<\frac{\beta-\alpha^{2}}{\beta \alpha^{2} \varphi(1-\mu)}
$$

is always verified for the parameter values in the range of interest. By comparing Eqs. (10) and (11) we obtain that if and only if

$$
\tau_{f}^{*}>\tau_{c 1}^{*} \quad \text { iff } \quad \gamma<\frac{\beta-\alpha^{2}}{\beta \alpha^{2}} \equiv \hat{\gamma},
$$

where $\hat{\gamma} \in\left[\hat{\gamma}_{c}, \hat{\gamma}_{f}\right]$. Finally, by comparing Eq. (10) and (12) we obtain that $\tau_{f}^{*}>\tau_{c 2}^{*}$.

We then inspect all the possible coalitions for different values of $\gamma$. For instance, for low levels of generosity $\gamma<\hat{\gamma}_{c}$, preferred tax rates are $\tau_{m}^{*}=\tau_{c 1}^{*}=0, \tau_{c 2}^{*}>0$ and $\tau_{f}^{*}>0$, with $\tau_{f}^{*}>\tau_{c 2}^{*}$. Positive tax rates result only if females and two-breadwinner couples form a majority (i.e. $2 \varphi \mu+(1-\varphi)>1$, which simplifies to $\mu>1 / 2$ ). In this case the smallest value of preferred tax rate $\tau_{c 2}^{*}$ is chosen because in a pairwise comparison between the two positive preferred tax rates, single males and one-breadwinner couples will vote for the smallest one.

\section{Model with continuous productivity distribution}

\section{C.1. Preferred tax rates}

Substituting for

$$
\frac{d p(\tau)}{d \tau}=\frac{1}{3} \frac{(1-2 \tau)}{\pi} \chi(\alpha, \beta, \varphi, \mu, \gamma)
$$


into Eqs. (B.1), (B.2), (B.3) and (B.4), we obtain that

$$
\left.\frac{\partial V^{f}(\tau)}{\partial \tau}\right|_{\tau=0}<0 \text { iff } \frac{\alpha^{2} w^{2}}{1 / 3}>\beta \chi(\alpha, \beta, \varphi, \mu, \gamma)
$$

Then, $\tau_{f}^{*}=0$. On the contrary, if $3 \alpha^{2} w^{2}<\beta \chi(\alpha, \beta, \varphi, \mu, \gamma)$, the preferred tax rate is positive and such that $\partial V^{f}\left(\tau_{f}^{*}\right) / \partial \tau=0$. In this case,

$$
\tau_{f}^{*}=\frac{\frac{\beta}{3} \chi(\alpha, \beta, \varphi, \mu, \gamma)-\alpha^{2} w^{2}}{2 \frac{\beta}{3} \chi(\alpha, \beta, \varphi, \mu, \gamma)-\alpha^{2} w^{2}}
$$

Using the same procedure for single men, we have that $\partial V^{m}(\tau) /\left.\partial \tau\right|_{\tau=0}<0$ if $3 w^{2}>\chi(\alpha, \beta, \varphi, \mu, \gamma)$ so that in this case, $\tau_{m}^{*}=0$, while for $3 w^{2}<\chi(\alpha, \beta, \varphi, \mu, \gamma), \tau_{m}^{*} \in[0,1]$ and is equal to (21). For two-breadwinner couples, $\partial V^{c 2}(\tau) /\left.\partial \tau\right|_{\tau=0}<0$ if $3 w^{2}>(1+\beta) \chi(\alpha, \beta, \varphi, \mu, \gamma) /$ $\left(1+\alpha^{2}\right)$; otherwise, it is equal to Eq. (14). For one-breadwinner couples, $\partial V^{c 1}(\tau) /\left.\partial \tau\right|_{\tau=0}<0$ if $3 w^{2}>(1+\gamma \beta) \chi(\alpha, \beta, \varphi, \mu, \gamma)$; otherwise the solution is interior and equal to Eq. (15).

\section{C.2. Equilibrium tax rate}

Substituting the expressions for $w_{f}\left(\tau^{*}\right), w_{c 2}\left(\tau^{*}\right)$ and $w_{c 1}\left(\tau^{*}\right)$ into Eq. (17) we get

$$
w_{m}\left(\tau^{*}\right)\left[(1-\varphi)\left(1+\frac{\sqrt{\beta}}{\alpha^{2}}\right)+2 \varphi \mu \sqrt{\frac{1+\beta}{1+\alpha^{2}}}+2 \varphi(1-\mu) \sqrt{1+\gamma \beta}\right]=1 .
$$

Denoting the term in brackets by $\Omega(\alpha, \beta, \varphi, \mu, \gamma)$, plugging the expression for $w_{m}\left(\tau^{*}\right)$, and rearranging, we obtain Eq. (18).

\section{C.3. Comparative statics}

Using Eq. Eq. (18), we obtain that

$$
\frac{\partial \tau^{*}}{\partial \gamma}=\frac{3 \Omega(.)\left[\frac{\partial \chi(.)}{\partial \gamma} \Omega(.)+2 \frac{\partial \Omega(.)}{\partial \gamma} \chi(.)\right]}{\left[2 \chi(.) \Omega(.)^{2}-3\right]^{2}}
$$

so that

$$
\frac{\partial \tau^{*}}{\partial \gamma}>0 \Leftrightarrow \frac{\partial \chi(.)}{\partial \gamma} \Omega(.)+2 \frac{\partial \Omega(.)}{\partial \gamma} \chi(.)>0 .
$$

Note that a similar expression holds for any parameter. What matters is the particular expression for the effect of the particular parameter on $\chi($.$) and \Omega($.$) and what final sign results when combined in the expression above.$

We have that

$$
\frac{\partial \chi(.)}{\partial \gamma}=-\frac{\left[1+\alpha^{2}(1-\varphi+\varphi \mu)\right] \beta \varphi(1-\mu)}{[1+\beta(1-\varphi+\varphi \mu+\gamma \varphi(1-\mu))]^{2}}=-\frac{\beta \varphi(1-\mu) \chi(.)}{1+\beta(1-\varphi+\varphi \mu+\gamma \varphi(1-\mu))}<0
$$

and

$$
\frac{\partial \Omega(.)}{\partial \gamma}=2 \varphi(1-\mu) \beta \frac{1}{2 \sqrt{1+\gamma \beta}}=\frac{\beta \varphi(1-\mu)}{\sqrt{1+\gamma \beta}}>0 .
$$

Combining in the expression above:

$$
\begin{aligned}
\frac{\partial \chi(.)}{\partial \gamma} \Omega(.)+2 \frac{\partial \Omega(.)}{\partial \gamma} \chi(.) & =-\frac{\beta \varphi(1-\mu) \chi(.)}{1+\beta(1-\varphi+\varphi \mu+\gamma \varphi(1-\mu))} \Omega(.)+2 \frac{\beta \varphi(1-\mu)}{\sqrt{1+\gamma \beta}} \chi(.) \\
& =\beta \varphi(1-\mu) \chi(.)\left[-\frac{\Omega(.)}{1+\beta(1-\varphi+\varphi \mu+\gamma \varphi(1-\mu))}+\frac{2}{\sqrt{1+\gamma \beta}}\right]
\end{aligned}
$$


The condition for a positive effect is

$$
\frac{\partial \tau^{*}}{\partial \gamma}>0 \Leftrightarrow \frac{1+\beta(1-\varphi+\varphi \mu+\gamma \varphi(1-\mu))}{\sqrt{1+\gamma \beta}}>\frac{\Omega(.)}{2} .
$$

This holds for reasonable values of $\mu, \varphi, \gamma \in[0,1]$ and also when $\beta$ increases. The only case in which the condition may not be satisfied is for $\alpha \leq 0.4$.

Using the same procedure for $\mu$, we have that

$$
\frac{\partial \tau^{*}}{\partial \mu}>0 \Leftrightarrow \frac{\partial \chi(.)}{\partial \mu} \Omega(.)+2 \frac{\partial \Omega(.)}{\partial \mu} \chi(.)>0,
$$

where

$$
\frac{\partial \chi(.)}{\partial \mu}=\varphi \frac{\alpha^{2}-\beta+\beta \gamma+\alpha^{2} \beta \gamma}{[1+\beta(1-\varphi+\varphi \mu+\gamma \varphi(1-\mu))]^{2}}
$$

and

$$
\frac{\partial \Omega(.)}{\partial \mu}=2 \varphi\left(\sqrt{\frac{1+\beta}{1+\alpha^{2}}}-\sqrt{1+\gamma \beta}\right)
$$

We can show that:

$$
\frac{\partial \chi(.)}{\partial \mu}>0 \Leftrightarrow \alpha^{2}-\beta+\beta \gamma\left(1+\alpha^{2}\right)>0 \Leftrightarrow \gamma>\frac{\beta-\alpha^{2}}{\beta\left(1+\alpha^{2}\right)}=\hat{\gamma}_{c},
$$

and

$$
\frac{\partial \Omega(.)}{\partial \mu}>0 \Leftrightarrow \frac{1+\beta}{1+\alpha^{2}}>1+\gamma \beta \Leftrightarrow \gamma<\frac{\beta-\alpha^{2}}{\beta\left(1+\alpha^{2}\right)}=\hat{\gamma}_{c} .
$$

For $\beta=1.2$ and $\alpha=0.8$, we obtain $\hat{\gamma}_{c}=0.2845$.

\section{References}

Bommier, A., Magnac, T., Rapoport, B., Roger, M., 2006. Droit à la retraite et mortalité différentielle. Economie et Prévision 168, 1-16.

Bonnet, C., Geraci, M., 2009. Comment corriger les iné galités de retraite entre hommes et femmes? L'expérience de cinq pays européens. Population et sociétés, Bulletin mensuel INED.

Borck, R., 2007. On the choice of public pensions when income and life expectancy are correlated. Journal of Public Economic Theory 9, $711-725$.

Browning, E., 1975. Why the social insurance budget is too large in a democracy. Economic Inquiry 13, 373-388.

Casamatta, G., Cremer, H., Pestieau, P., 2000. The political economy of Social Security. Scandinavian Journal of Economics $102,503-522$.

Choi, J., 2006. The role of derived rights for old-age income security of women. Social Employment and Migration. Working Paper 43. OECD, Paris.

Coronado, J.L., Fullerton, D., Glass, T., 2000. The progressivity of Social Security. Working Paper 7520. NBER, Cambridge (MA).

de Walque, G., 2005. Voting on pensions: a survey. Journal of Economic Surveys 19, 181-209.

Galasso, V., 2002. Social Security: a financial appraisal for the median voter. Social Security Bulletin 64, 57-65.

Galasso, V., Profeta, P., 2002. The political economy of Social Security: a survey. European Journal of Political Economy 18, 1-29.

Gruber, J., Wise, D., 1999. Social Security and Retirement around the World. Chicago University Press, Chicago.

Leroux, M.-L., 2010. The political economy of Social Security under differential longevity and voluntary retirement. Journal of Public Economic Theory $12,151-170$. Leroux, M.-L., Pestieau, P., 2010. The political economy of derived pension rights. Discussion Paper 48. CORE, Louvain-la Neuve.

Liebman, J.B., 2001. Redistribution in the current U.S Social Security system. Working Paper 8625. NBER, Cambridge (MA).

Mare, R., 1991. Five decades of educational assortative mating. American Sociological Review 56, 15-32.

Pencavel, J., 1998. Assortative mating by schooling and the work behavior of wives and husbands. The American Economic Review 88, $326-329$.

Qian, Z., 1998. Changes in assortative mating: the impact of age and education, 1970-1990. Demography 35, $279-292$.

Recoules, M., 2009. How can gender discrimination explain fertility behaviors and family-friendly policies?. Mimeo.

Veil, M., 2007. L'individualisation des droits dans l'assurance vieillesse: débats et axes de réformes. Retraite et soci été 50, 115-126. 